\title{
Coherent Multiple Scattering Effects and Monte Carlo Method
}

\author{
V. L. Kuzmin ${ }^{1} *$ and I. V. Meglinski² \\ ${ }^{1}$ St. Petersburg Institute of Commerce and Economics, ul. Novorossiüskaya 50, St. Petersburg, 194021 Russia \\ ${ }^{2}$ Cranfield University, School of Engineering, Cranfield, MK43 OAL, United Kingdom \\ *e-mail: vladimir.kuzmin@paloma.spbu.ru
}

\begin{abstract}
Based on the comparison of the iteration procedure of solving the Bethe-Salpeter equation and the Monte Carlo method, we developed a method for simulating coherent multiple-scattering effects within the framework of a unified stochastic approach. The time correlation function and the interference component were calculated for the coherent backscattering from a multiply scattering medium.
\end{abstract}

PACS numbers: 42.25.Fx; 11.10.St; 02.70.Uu

Numerical simulation based on the stochastic Monte Carlo method is widely used to calculate the intensity of scattered radiation in randomly inhomogeneous turbid media [1]. However, a special approach is required to apply the method for simulating coherent effects, which manifest themselves even in the presence of strong multiple scattering and have attracted increasing interest in recent years [2-8].

In this work, the stochastic Monte Carlo method is compared with a theoretical approach based on the representation of the Bethe-Salpeter equation in the form of a multiple scattering series to show how this method is generalized within the framework of a unified stochastic approach for calculating time correlations of intensity, coherent backscattering, and other coherent effects.

The field correlation function in an inhomogeneous dispersive medium with random space-time fluctuations of the dielectric constant is described by the integral Bethe-Salpeter equation

$$
\begin{gathered}
\Gamma\left(\mathbf{R}_{2}, \mathbf{R}_{1}, t \mid \mathbf{k}_{\mathrm{s}}, \mathbf{k}_{\mathrm{i}}\right)=\mu p_{t}\left(\mathbf{k}_{\mathrm{i}}-\mathbf{k}_{\mathrm{s}}\right) \delta\left(\mathbf{R}_{2}-\mathbf{R}_{1}\right) \\
+\mu \int p_{t}\left(\mathbf{k}_{23}-\mathbf{k}_{\mathrm{s}}\right) \Lambda\left(R_{23}\right) \Gamma\left(R_{3}, \mathbf{R}_{1}, t \mid \mathbf{k}_{31}, \mathbf{k}_{\mathrm{i}}\right) d \mathbf{R}_{3} .
\end{gathered}
$$

Here, the Green's function (propagator) $\Gamma\left(\mathbf{R}_{2}, \mathbf{R}_{1}, t \mid \mathbf{k}_{\mathrm{s}}\right.$, $\mathbf{k}_{\mathrm{i}}$ ) of the Bethe-Salpeter equation describes the propagation of a pair of time-shifted (by $t$ ) complex conjugate fields from the point $\mathbf{R}_{1}$ with the incident wave vector $\mathbf{k}_{\mathrm{i}}$ to the point $\mathbf{R}_{2}$ with the outgoing wave vector $\mathbf{k}_{\mathrm{s}} ; k_{\mathrm{s}}=k_{\mathrm{i}}=k=n k_{0}$, where $k_{0}=2 \pi / \lambda$ is the wavenumber, $\lambda$ is the wavelength; $n=n_{1}+i n_{2}$ is the refractive index of a medium; and $\left(2 n_{2} k_{0}\right)^{-1}=l$ is the photon mean free path. For simplicity, we restrict ourselves only to the case where the intrinsic absorption is absent and light losses are caused only by elastic scattering. In this case, the scattering coefficient is $\mu=l^{-1}$. The function $\Lambda(R)=$ $R^{-2} \exp (-R / l)$ is the product of a complex conjugate pair of Green's functions of the corresponding wave equation and describes the radiation propagation between two scattering events. The function $p_{t}\left(\mathbf{k}_{\mathrm{i}}-\mathbf{k}_{\mathrm{s}}\right)$ is expressed in terms of the Fourier transform of the pair correlation function of dielectric constant fluctuations and coincides at $t=0$ with the scattering phase function $p_{0}\left(\mathbf{k}_{\mathrm{i}}-\mathbf{k}_{\mathrm{s}}\right)=p\left(\mathbf{k}_{\mathrm{i}}-\mathbf{k}_{\mathrm{s}}\right)$.

Let a scattering medium occupy the half-space $z>0$, where $z$ is the Cartesian coordinate normal to the medium boundary. For the normal incidence and backscattering, the incoherent component of the time correlation function is described by the ladder-diagram series and has the form

$$
\begin{gathered}
C^{(L)}\left(t \mid \mathbf{k}_{\mathrm{s}}, \mathbf{k}_{\mathrm{i}}\right)=\int d \mathbf{R}_{1} d \mathbf{R}_{2} \\
\times \Gamma\left(\mathbf{R}_{2}, \mathbf{R}_{1}, t \mid \mathbf{k}_{\mathrm{s}}, \mathbf{k}_{\mathrm{i}}\right) \exp \left[-\mu\left(z_{1}+z_{2}\right)\right] .
\end{gathered}
$$

For scattering angles $\theta_{\mathrm{s}}$ close to $180^{\circ}$, the interference component caused by the cyclic $[9,10]$ or fan diagrams is comparable with the ladder component:

$$
\begin{gathered}
C^{(V)}\left(t \mid \mathbf{k}_{\mathrm{s}}, \mathbf{k}_{\mathrm{i}}\right)=\int d \mathbf{R}_{1} d \mathbf{R}_{2} \\
\times\left[\Gamma\left(\mathbf{R}_{2}, \mathbf{R}_{1}, t \mid \frac{\mathbf{k}_{\mathrm{s}}-\mathbf{k}_{\mathrm{i}}}{2}, \frac{\mathbf{k}_{\mathrm{i}}-\mathbf{k}_{\mathrm{s}}}{2}\right)\right. \\
\left.-\mu p_{t}\left(\mathbf{k}_{\mathrm{i}}-\mathbf{k}_{\mathrm{s}}\right) \delta\left(\mathbf{R}_{2}-\mathbf{R}_{1}\right)\right] \\
\times \exp \left[-\mu\left(z_{1}+z_{2}\right)+i k_{0}\left(x_{1}-x_{2}\right) \sin \theta_{s}\right] .
\end{gathered}
$$

The incident and scattered beams lie in the $(x, z)$ plane. 
Intensity of backscattered light as a function of the layer thickness $L$ for various anisotropy parameters

\begin{tabular}{l|c|c|c|c|c}
\hline$\overline{\cos \theta}$ & $L=l^{*}$ & $L=2 l^{*}$ & $L=5 l^{*}$ & $L=10 l^{*}$ & $\infty$ \\
\hline 0 & 0.3481 & 0.5254 & 0.7507 & 0.8665 & 1 \\
0.9 & 0.3214 & 0.5318 & 0.7784 & 0.9003 & 1 \\
\hline
\end{tabular}

At $t=0$, Eq. (2) describes the backscattering intensity. Correspondingly, Eq. (3) determines the peak in the coherent backscattering.

By integrating the Bethe-Salpeter equation, one arrives at a series that is usually illustrated by a series of ladder diagrams.

Let us compare the analytic procedure of summing ladder-diagram series with the Monte Carlo method. The first term of the iterative series describes single scattering, the second term describes two scattering events, etc. Similarly, the Monte Carlo method describes the radiation propagation as a stochastic process consisting of $1,2, \ldots, N$ scattering events. The addition of one ladder section $\Lambda\left(R_{n n+1}\right) p_{0}\left(\mathbf{k}_{n n+1}-\right.$ $\left.\mathbf{k}_{n+1}\right)$ in the theoretical description is realized in the numerical experiment by modeling the photon paths through a certain distance $s$ to the next scattering event. The main assumption in the stochastic Monte Carlo method consists of postulating the distribution law $f(s)=\mu \exp (-\mu s)$ for the photon mean free path as a random variable $s$ [11]. It follows from this distribution that $s=-\mu^{-1} \ln \xi$, where $\xi$ is the probability that the mean free path is no less than $s$. In the Monte Carlo method, the arbitrary $\xi$ value is chosen in the $[0,1]$ interval using a random number generator. The change in the direction of motion of the photon package in each elastic scattering event is determined by the scattering phase function.

Physically, the series arising upon the iteration of Eq. (1) is a series in scattering multiplicity. If the integration with respect to $R_{i}$ is replaced by a random choice $R_{i}=s$ and the integration with respect to $\mathbf{R}_{\mathrm{i}}$ is realized by a random choice of angles with the statistical weight determined by the phase function, then the solution of the Bethe-Salpeter equation is simulated by the Monte Carlo scheme described above, making it possible to use it for calculating coherent effects.

The majority of applications $[12,13]$ are devoted to the diffusion mechanism of inhomogeneity time evolution, for which the time correlation function of intensity fluctuations can be represented as the product of static correlation function and exponential

$$
p_{t}(q) \approx p_{0}(q) \exp \left(-D_{\mathrm{s}} q^{2} t\right),
$$

where $D_{\mathrm{s}}$ is the self-diffusion coefficient. The only difference between the calculation of the time correlation function and the calculation of intensity is that the weight of the photon package is multiplied by the phase function $p_{t}\left(\mathbf{k}_{n}-\mathbf{k}_{n-1}\right)$ in each scattering event.

The number of incident photons varied in the range $10^{5}-10^{7}$. The simulation of photon trajectory was terminated when the number of scattering events exceeded $10^{4}$. We also disregarded photons whose statistical weights became less than $10^{-3}$. This neglect leads to an error no higher than $10^{-5}$, because, according to our estimates, the probability of detecting such a photon on the surface is no higher than $10^{-2}$. The accuracy of calculated parameters was tested by the stability of numerical values upon increasing the sample size. For a number of $10^{5}$, the intensity is stable with an accuracy of no less than four decimal places.

The time correlation function of the field $g_{1}(t)$ was calculated for media with various anisotropy factors $\overline{\cos \theta}=0,0.5$, and 0.9. The Henyey-Greenstein phase function was used in simulation. In terms of $\sqrt{t / \tau}$, where $\tau=1 / D_{\mathrm{s}} k^{2}$ is the characteristic diffusion time of a scattering particle through a distance on the order of the wavelength, the time correlation function is virtually universal and is independent of the anisotropy of single scattering, in agreement with experiments [12, 13] and theoretical solution in the $P_{1}$ approximation [14], while the specificity of the scattering system is taken into account upon the transition to the description in units of characteristic time $\tau$. The dependence obtained for the time correlation function is well described by the formula $g_{1}(t) \propto \exp (-\gamma \sqrt{6 t / \tau})$ proposed in [13].

The time correlation function was calculated for layers of different thickness. The corresponding dependence on the average $\operatorname{cosine} \overline{\cos \theta}$ of the single-scattering angle is weak enough for finite-thickness layers, as is seen in the table, where $l^{*}=l(1-\overline{\cos \theta})^{-1}$ is the transport mean free path.

Figure 1 shows the results of simulation of the field time correlation functions for finite-thickness layers with $\overline{\cos \theta}=0.9$. As is seen, the diffusion character of light propagation with a linear dependence on $\sqrt{t / \tau}$ reveals itself at increasingly large times with a decrease in thickness. Good agreement with the experimental data is noteworthy: Fig. 1 almost exactly reproduces Fig. 2 from [13].

Figure 2 also shows the angular dependence of the coherent backscattering peak calculated for $\overline{\cos \theta}=0$, 0.5 , and 0.9. It follows from Eq. (3) that, in the case of normal incidence and small backscattering angles, the difference from the expression for the intensity of the incoherent component consists only in the presence of the additional factor $\exp \left(i \mathbf{q}_{\perp}\left(\boldsymbol{\rho}_{1}-\boldsymbol{\rho}_{2}\right)\right)$, which can be replaced by $\cos \left(\mathbf{q}_{\perp}\left(\boldsymbol{\rho}_{1}-\boldsymbol{\rho}_{2}\right)\right)$ because of the translational invariance about the transverse coordinates $\boldsymbol{\rho}$. 


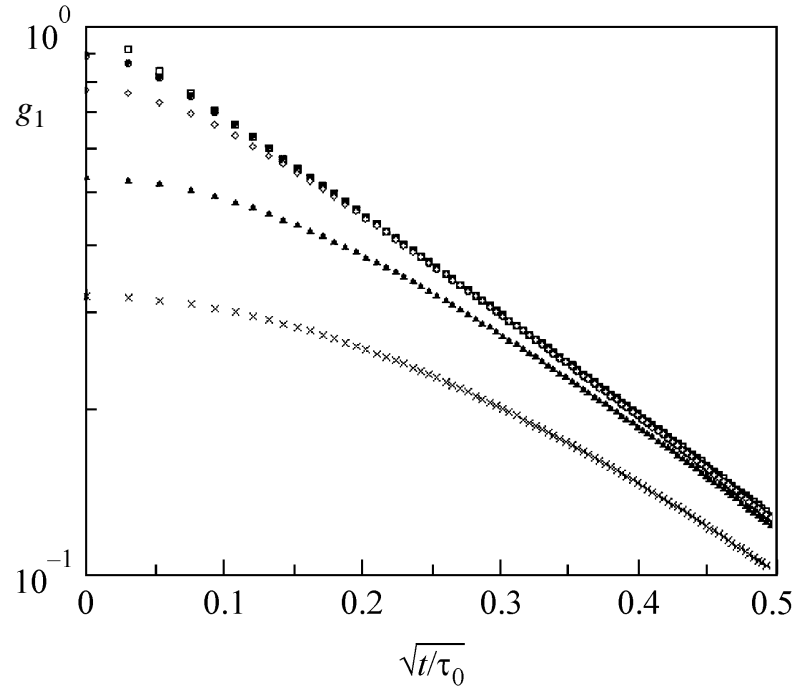

Fig. 1. Time correlation functions $g_{1}$ of backscattered radiation for layers with thickness $L=(\times) l^{*},(\boldsymbol{\Delta}) 2 l^{*},(\diamond) 5 l^{*}$, (•) $10 l^{*}$, and $(\square) \infty$ (semi-infinite medium). All data are normalized to the intensity of light diffusively reflected from the semi-infinite medium. The optical parameters of the medium are $\mu=30 \mathrm{~mm}^{-1}$ and $\overline{\cos \theta}=0.9$.

Then, when calculating the intensity of the backscattering coherent component, one should, first, multiply the total weight of photon packages arriving with vector $\mathbf{k}_{\mathrm{s}}$ at distance $\rho$ from the entry point at the interface by the factor $\cos \left(\mathbf{q}_{\perp} \boldsymbol{\rho}\right)$ and, second, take the sum over the entire surface.

Similarly to the time correlations, the angular dependence of the coherent backscattering peak intensity proves to be universal in the dimensionless variable $\tilde{q}=k l^{*} \sin \theta_{s}$ and is well described by the formula $I_{\mathrm{CBS}} \propto \exp \left(-\gamma k l^{*} \sin \theta_{s}\right)$ with $\gamma=2$. Note that the obtained universal dependence $I_{\mathrm{CBS}} \propto \exp \left(-\gamma k l^{*} \sin \theta_{s}\right)$ with $\gamma=2$ differs significantly from the dependence [15]

$$
I_{\mathrm{CBS}}^{\mathrm{diff}} \propto 1-2 \frac{\left(1+z^{*}\right)^{2}}{1+2 z^{*}} k l^{*} \sin \theta_{s},
$$

predicted in the diffusion approximation for $k l^{*} \sin \theta_{s} \ll$ 1 , where $z^{*}=0.71(1-\overline{\cos \theta})^{-1}$. This formula gives the slope $\gamma^{\text {(diff) }}=2.3$ for $\overline{\cos \theta}=0$ and $\gamma^{\text {(diff) }}=0.71$ for $\overline{\cos \theta} \longrightarrow 1$.

Contrary to the diffusion approximation, which predicts that the linear slope of the coherent backscattering peak decreases with an increase in anisotropy, the dependence calculated by us indicates the universal character of a decrease. If the phase function is strongly anisotropic, the coherence effects can be quite pronounced, despite the smallness of these parameters. In particular, the decay of intensity time correlations is governed by the parameter $t / \tau$, which can be much

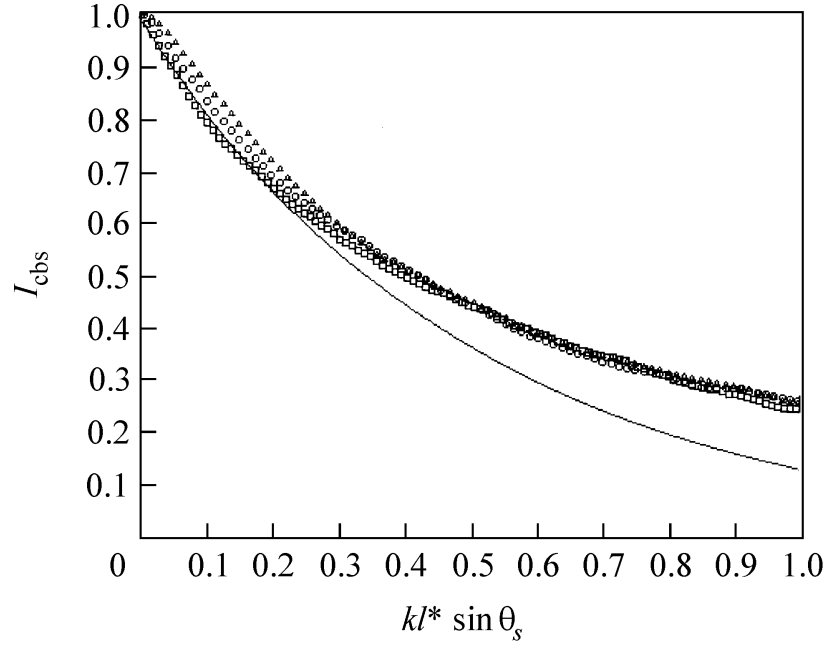

Fig. 2. Peak intensity of coherent backscattering vs. the dimensionless parameter $k l^{*} \sin \theta_{s} ; \lambda=0.6 \mu \mathrm{m} ; n_{1}=1 ; \mu=$ $30 \mathrm{~mm}^{-1} ; \overline{\cos \theta}=(\square) 0,(\bigcirc) 0.5$, and $(\triangle) 0.9$; and the solid line is the $\exp \left(-2 k l^{*} \sin \theta_{s}\right)$ approximation.

greater than the parameter $(t / \tau)\left(l / l^{*}\right)$. It is precisely due to this fact that the intensity correlation functions are described by the multiple-scattering theory even if they decrease by two orders of magnitude.

In this work, the coherent effects in multiple scattering have been simulated within the framework of a unified stochastic approach. Similar calculations can easily be carried out for suspensions that are usually treated as a system of hard spheres [16]. The phase function for this system is represented as the product of the Mie form factor and the Percus-Yevick structure factor.

The comparative analysis carried out in this work enables one to considerably simplify the simulation of radiation transport and coherent effects in randomly inhomogeneous strongly scattering media, such as liquid crystals, tissues, etc., and to extend the application field of these methods.

We are grateful to D. Yu. Churmakov for assistance in calculations and V.P. Romanov for valuable advice. This work was supported by the Russian Foundation for Basic Research (project no. 02-02-16577), the Royal Society (grant no. 15298), and NATO (grant no. PST.CLG.979652).

\section{REFERENCES}

1. V. P. Kandidov, Usp. Fiz. Nauk 166, 1309 (1996) [Phys. Usp. 39, 1243 (1996)].

2. M. Ospeck and S. Fraden, Phys. Rev. E 49, 4578 (1994).

3. T. Iwai, H. Furukawa, and T. Asakura, Opt. Rev. 2, 413 (1995).

4. K. Ishii, T. Iwai, and T. Asakura, Opt. Rev. 4, 643 (1997). 
5. S. E. Skipetrov and S. S. Chesnokov, Kvantovaya Élektron. (Moscow) 25, 753 (1998).

6. R. Lenke and G. Maret, Eur. Phys. J. B 17, 171 (2000).

7. S. E. Skipetrov and I. V. Meglinskiı̌, Zh. Éksp. Teor. Fiz. 113, 1213 (1998) [JETP 86, 661 (1998)].

8. R. Lenke, R. Tweer, and G. Maret, J. Opt. A: Pure Appl. Opt. 4, 293 (2002).

9. Yu. N. Barabanenkov, Izv. Vyssh. Uchebn. Zaved., Radiofiz. 16, 88 (1973).

10. A. A. Golubentsev, Zh. Éksp. Teor. Fiz. 86, 47 (1984) [Sov. Phys. JETP 59, 26 (1984)].

11. I. M. Sobol', The Monte Carlo Method (Nauka, Moscow, 1985).
12. G. Maret and P. Z. Wolf, Z. Phys. B 65, 409 (1987).

13. D. J. Pine, D. A. Weitz, P. M. Chaikin, and E. Herbolzheimer, Phys. Rev. Lett. 60, 1134 (1988).

14. V. L. Kuzmin, V. P. Romanov, and E. V. Aksenova, Phys. Rev. E 65, 016601 (2002).

15. E. Akkermans, P. E. Wolf, R. Maynard, et al., J. Phys. (Paris) 49, 77 (1988).

16. V. L. Kuz'min, V. P. Romanov, and I. V. Meglinskiĭ, Opt. Spektrosk. 96, 139 (2004) [Opt. Spectrosc. 96, 106 (2004)]. 